\title{
Construcción de un índice de riesgo para la infección por el VIH y su relación con la doble moral y el poder diádico en adolescentes
}

\author{
María P. Bermúdez*, María T. Ramiro, Juan C. Sierra y Gualberto Buela-Casal
}

Universidad de Granada (Granada, España)

\begin{abstract}
Resumen: El objetivo de este estudio fue analizar la relación entre el poder sobre la relación de pareja y la doble moral, y su influencia en la predicción del riesgo de infección de VIH en adolescentes colombianos. La muestra fue de 599 adolescentes (entre 12 y 18 años), de ambos sexos, estudiantes de secundaria de colegios públicos y privados. Para construir el índice de riesgo se utilizaron las siguientes variables (preguntando siempre sobre el período de los últimos 12 meses): Uso consistente del preservativo, número de parejas sexuales, tener relaciones sexuales bajo los efectos de alguna droga, haber utilizado la píldora del día después, e iniciativa en el uso del preservativo. Los predictores fueron el poder en la relación de pareja y la doble moral. Se obtuvo que las mujeres tienen un mayor poder en sus relaciones de pareja y menor adhesión a la doble moral. La baja adherencia a la doble moral se relacionó con un mayor poder en la relación y éste predijo un menor riesgo de infección por VIH (la doble moral no se relacionó directamente con riesgo de infección de VIH). En varones la adherencia a la doble moral predice un mayor riesgo de infección.

Palabras clave: Relaciones de poder en la pareja; doble moral; VIH; adolescentes.
\end{abstract}

\section{Introducción}

Un gran número de estudios sobre el uso consistente del preservativo en adolescentes concluyen que las mujeres informan de un menor uso del mismo (Eaton et al., 2010; Fagan y McDonell, 2010; Gayet, Juárez, Pedrosa y Magis, 2003; Gurman y Borzekowski, 2004; Teva, Bermúdez y BuelaCasal, 2009). Una posible explicación a este hecho es que las mujeres tienen menor poder en sus relaciones de pareja (Fernández-Esquer, Atkinson, Diamond, Useche y Mendiola, 2004; Harvey, Beckman, Browner, y Sherman, 2002). A su vez, este bajo poder en las relaciones se explica por el hecho de que, en sociedades patriarcales, se espera que el varón domine las relaciones sociales y sexuales y que la mujer se muestre sumisa, lo que, junto con evaluar conductas sexuales como inmorales o no en función del sexo del emisor de dichas conductas, se conoce como doble moral (Dávila, 2002; Greig, Peacock, Jewkes y Msimang, 2008).

Una de las primeras investigaciones realizada para evaluar la relación entre la adhesión a la doble moral y el uso consistente del preservativo es el estudio de Noar y Morokoff (2002). Los autores analizaron la relación entre la ideología masculina y la intención de usar el preservativo, concluyendo que la adhesión a la ideología masculina correlaciona con actitudes negativas hacia el uso del preservativo, y éstas predicen una menor intención de uso. Sin embargo, el poder en la relación no se evalúa. Otras investigaciones han llegado a resultados similares, al relacionar una doble moral elevada con

* Dirección para correspondencia [Correspondence address]: MâPaz Bermúdez. Facultad de Psicología. Universidad de Granada. 18011-Granada (España) E-mail: maripaz@ugr.es

\begin{abstract}
Title: Construction of a risk index of HIV infection and its relation to double morale and dyadic power in adolescents

Abstract: The current study aimed to analyze the link between power over couple relationship and double morale, and its influence on the prevention of HIV infection risk in Colombian adolescents. The sample was composed of 599 adolescents, males and females aged between 12 and 18 studying in public or private schools. In order to construct the risk index the following variables were used: Consistent condom use, Number of sexual partners, having sexual intercourse under the effects of some drug, Having used the morning-after pill and Self-initiative in condom use. Predictors were power over couple relationship and double morale. Results indicated that women had more power in their couple relationships and lower adherence to double morale. Low adherence to double morale was related to more power in the relationship, which predicted less risk of HIV infection. Double morale was not directly associated with risk of HIV infection. In men, adherence to double morale predicted an enhanced risk of infection. Key words: Power relationships in couple; double morale; adolescents.
\end{abstract}

mayor número de parejas sexuales (Kreager y Staff, 2009; Zangao y Sim-Sim, 2011).

Posteriormente, se han realizado estudios en los que se analiza la doble moral sexual y el poder en la relación, con medidas directas de la primera. Por ejemplo, Bermúdez, Castro, Gude y Buela-Casal (2010) concluyen que los varones muestran una mayor doble moral que las mujeres y que éstas tienen mayor poder en la relación. Además, Bowleg, Belgrave y Reisen (2000) concluyen que la adhesión a un rol tradicional predice un menor poder en la relación y éste se vincula con una menor autoeficacia en el uso del preservativo. Sin embargo, la muestra era de mujeres, por ello, no se puede conocer cómo influye la doble moral y el poder sobre la relación, en varones. Este aspecto es importante ya que existe un debate entre investigadores sobre si la doble moral tiene su origen en las actitudes de los varones o de las mujeres. Desde el enfoque que defiende que las mujeres tienen mayor adhesión a la doble moral se afirma que éstas tendrían mayor poder sobre la relación, pero lo utilizarían para evitar la actividad sexual (Baumeister y Twenge, 2002). Prueba de ello, es que las mujeres, con alta adhesión a la doble moral, con mayor frecuencia se mantienen abstinentes sexualmente y son más reacias a hablar de aspectos relacionados con la sexualidad (Jackson y Cram, 2003; Leftkowitz, Boone y Shearer, 2004). Desde la hipótesis contraria, el varón sería el que forzaría a la mujer a adoptar prácticas sexuales desprotegidas (Dávila, 2002; Greig et al., 2008). Pulerwitz, Amaro, De Jong, Gortmaker y Rudd (2002) indican que las mujeres con mayor poder sobre la relación de pareja presentan una mayor probabilidad de hacer un uso consistente del preservativo. Según esto, y de ser cierto que las mujeres son las que mantienen la doble moral, un mayor poder en la relación predeciría un menor riesgo de infección por VIH. En cambio, si 
fuese el varón el que mantiene la doble moral, ésta predeciría un mayor riesgo de infección (Pulerwitz et al., 2002).

Por último, hay evidencias de que los inmigrantes latinos son un colectivo de riesgo para las ITS y el VIH (Bermúdez, Castro y Buela-Casal, 2009; Bermúdez, Castro, Madrid y Buela-Casal, 2010; Castro y Bermúdez, 2011; Motti-Stefanidi et al., 2008) y la doble moral puede ser uno de los factores que influyan en este hecho. Por ello, es necesario estudiar si hay diferencias transculturales en la doble moral. Así pues, el objetivo de este estudio (con un diseño ex-post-facto retrospectivo factorial; Montero y León, 2007) es analizar las relaciones que existen entre los constructos poder sobre la relación de pareja y doble moral, y su influencia en la predicción del riesgo de infección de VIH en varones y mujeres adolescentes, residentes en Colombia.

\section{Método}

\section{Participantes}

La muestra estaba compuesta por 599 alumnos de Secundaria de Colombia, tanto de colegios públicos (46.24\%) como privados, con edades entre 12 y 18 años $(M=15.58$, $D T=1.41)$ y que habían tenido al menos una relación sexual. La edad media de los varones fue de 15,48 (DT $=$ $1,41)$ y de las mujeres de $15,76(D T=1,40)$. El $65.94 \%(395$ personas) eran varones. Un $14.99 \%$ eran practicantes de su religión, un $63.03 \%$ algo practicantes y un $21.98 \%$ no practicantes. La selección fue incidental dentro de los centros, estratificando las cotas de sexo y edad para balancear la muestra.

\section{Instrumentos}

Para construir el índice de riesgo, de acuerdo con Kotchick, Shaffer, Forehand y Miller (2001), se utilizaron variables que hacen referencia a aspectos conductuales situados a un mismo nivel de abstracción e interrelación:

Uso consistente del preservativo en los últimos 12 meses. Se diferenció su uso según tipo de pareja (estable y ocasional) y tipo de actividad sexual (vaginal, oral y anal), en los últimos 12 meses. El uso consistente del preservativo es mayor en las parejas ocasionales (Piña-López, Lozano-Ramírez, VázquezRamírez y Carrillo-Saucedo, 2010), y mayor en las relaciones sexuales vaginales frente a las anales, en heterosexuales (Gurman y Berzekowski, 2004; Robles-Montijo, Piña-López y Moreno-Rodríguez, 2006). También se preguntó por el uso del preservativo en su debut sexual, ya que ello predice el uso posterior del preservativo (Gayet et al., 2003; MorenoRodríguez et al., 2008; Noar y Morokoff, 2002).

El uso consistente del preservativo en parejas estables y en parejas ocasionales se estimó a partir de un ítem, sobre la frecuencia de uso del preservativo ("Siempre", "Algunas veces" y "Nunca”). Se consideró que usaba consistentemente el preservativo si se respondía "Siempre".
Respecto al uso del preservativo en función del tipo de actividad sexual, en los tres casos (vaginal, anal y oral) se preguntó sobre la frecuencia con la que se daba esta actividad y la frecuencia con la que se utilizaba el preservativo, en ambos casos durante los últimos 12 meses, mediante las alternativas "Nunca", "Una vez", "Cerca de una vez al mes", "Cerca de una ver a la semana" y "Varias veces a la semana". Se consideró que se usaba consistentemente el preservativo cuando se utilizaba con la misma frecuencia con la que se tenía relaciones sexuales.

Número de parejas sexuales en los últimos 12 meses. Es una variable muy utilizada para estimar índices de riesgo (Gurman y Borzekowski, 2004; Organista, Organista, Bola, García-deAlba y Castillo-Morán, 2000; Teva, Bermúdez y Buela-Casal, 2011). Teóricamente, a mayor número de parejas sexuales aumenta la probabilidad de tener relaciones sexuales con una persona infectada de VIH (Gurman y Borzekowski, 2004).

Esta variable fue dicotomizada como en Organista et al. (2000): no haber tenido ninguna pareja sexual en los últimos 12 meses (no riesgo) y haber tenido pareja sexual (riesgo).

Tener relaciones sexuales bajo los efectos de alguna droga en los últimos 12 meses. Esta variable ha sido relacionada con conductas sexuales de riesgo (Antón-Ruiz y Espada, 2009; Gurman y Borzekowski, 2004; Kalichman, Eaton y Cherry, 2010).

Tener relaciones sexuales bajo los efectos de las drogas se evaluó mediante un ítem que recogía el alcohol y las drogas inhaladas e inyectadas, más frecuentes en Colombia. Se contabiliza si hubo uso de, al menos, una de las sustancias en los últimos 12 meses como indicador de riesgo.

Haber utilizado la píldora del día después en los últimos 12 meses. Este indicador ha sido utilizado en varios modelos de predicción de conductas sexuales de riesgo (Phipps, Matteson, Fernández, Chiaverini y Weitzen, 2008; Sander, Raymond y Weaver, 2009). Se evaluó mediante un ítem.

Iniciativa en el uso del preservativo en los últimos 12 meses. Los estudios que utilizan esta variable (Gayet et al., 2003) encuentran que la iniciativa es mucho mayor en varones que en mujeres.

Escala de Poder en las Relaciones de Pareja (EPRP; Pulerwitz, Gortmaker y DeJong, 2000). Este instrumento permite evaluar el grado en que uno de los miembros de la pareja tiene más poder y control sobre las decisiones que afectan, entre otras, a la actividad sexual o al uso del preservativo en las relaciones sexuales (Pulerwitz et al., 2002). La versión final está compuesta por 23 ítems que evalúan dos subescalas: control sobre la relación (15 ítems [por ejemplo, "Mi pareja hace lo que él/ella quiere, aun si yo no quiero que lo haga"], con cuatro alternativas de respuesta, desde "Muy de acuerdo" hasta "Muy en desacuerdo") y control sobre la toma de decisiones (8 ítems [por ejemplo, “¿Quién tiene normalmente mayor peso acerca de qué cosas hacen ustedes juntos"], con tres alternativas de respuesta: "Su pareja", "Ambos por igual" y "Usted").

Únicamente en la subescala de control sobre la relación se obtuvo una adecuada consistencia interna (original de .88; 
presente muestra de .82). Las correlaciones ítem-total corregidas oscilaron de .25 a .56. De acuerdo con Muñiz, Hidalgo, Cueto, Martínez y Moreno (2005) el ítem 15 no discriminaba adecuadamente. Se mantuvo por su bajo efecto sobre la media, varianza y consistencia interna de la subescala. Como índice resumen se adoptará la media de los valores de los ítems (valores de 1 a 4 ).

Escala de Doble Moral (EDM; Caron, Davis, Halterman y Stickle, 1993). Se usó la adaptación salvadoreña de Sierra y Gutiérrez-Quintanilla (2007). Diseñada para evaluar el grado de aceptación de una moral más permisiva a la hora de juzgar la conducta sexual de los varones que de las mujeres (Caron et al., 1993; Jackson y Cram, 2003). Está compuesta por 10 ítems (por ejemplo, "En el sexo, el varón debe tomar el rol dominante y la mujer el rol pasivo") con cinco alternativas de respuesta (desde "Muy en desacuerdo" hasta "Muy de acuerdo").

En la versión salvadoreña (Sierra y Gutiérrez-Quintanilla, 2007), se obtuvo una consistencia interna de .78, cuando se eliminaban los ítems 8 y 3 . En el presente estudio la consistencia interna fue de .74, tras la eliminación del ítem 8 (baja correlación ítem-total corregida). Como índice resumen de la puntuación en la escala se adoptó la media (por tanto, entre 1 y 5).

\section{Procedimiento}

El cuadernillo definitivo se pasó a varios expertos para que evaluasen posibles dificultades de comprensión en adolescentes colombianos. Tras las modificaciones indicadas por los expertos, se contactó incidentalmente con centros educativos para presentar el estudio. La aplicación se llevó a cabo de forma colectiva, en las propias clases, de una media de 30 alumnos, en horario lectivo en un tiempo medio de 35 minutos. Junto con el cuadernillo se entregó una hoja de consentimiento informado.

\section{Resultados}

\section{Construcción del índice global de riesgo de infec- ción por el VIH}

Los porcentajes de las alternativas que implican riesgo para la infección por el VIH, de los ítems utilizados para construir el índice global de riesgo se pueden consultar en la Tabla 1. Según el modelo de Rasch (1960), conforme menor sea la proporción de participantes que seleccionen una alternativa, menor será su valor. Por tanto, los ítems que representan un mayor riesgo son los que tienen una puntuación menor. En la Tabla 1 se puede observar que la conducta de riesgo que tiene mayor ocurrencia $(60.16 \%$ de los participantes) es no utilizar el preservativo en las relaciones orales. En el otro extremo, la conducta menos realizada (19.01\%) es utilizar, durante el último año, la píldora del día después.

En la Tabla 1, aparecen diferentes índices de ajuste. Mediante la correlación biserial-puntual (item-total), se observa que los ítems 8 y 10 presentaron correlaciones muy bajas. Sin embargo, los estadísticos Infit y Outfit adoptaron valores comprendidos entre 0.7 y 1.3 (que representa los valores de corte para un 30\% de error; Wright y Masters, 1982, p. 100). Se produjeron sobreajustes en los ítems 3,4 y 6 . Sin embargo, este tipo de falta de ajuste tiene poca importancia (Wright y Linacre, 1988).

Tabla 1. Estadísticos de ajuste para los índices de riesgo de infección por VIH.

\begin{tabular}{|c|c|c|c|c|c|c|c|c|}
\hline \multirow{2}{*}{ Conductas de riesgo } & \multirow{2}{*}{$\%$} & \multirow{2}{*}{$\begin{array}{l}\text { Medida } \\
\text { de riesgo }\end{array}$} & \multirow{2}{*}{ SE } & \multicolumn{2}{|c|}{$I N F I T 1^{1}$} & \multicolumn{2}{|c|}{ OUTFIT $^{2}$} & \multirow{2}{*}{$\begin{array}{c}\text { Correlación } \\
\text { biserial-puntual }\end{array}$} \\
\hline & & & & $M N S Q^{3}$ & ZSTD 4 & $M N S Q^{3}$ & $Z S T D^{4}$ & \\
\hline 1. NUCP5 en relaciones sexuales orales & 60.16 & -0.77 & 0.10 & 0.97 & -0.6 & 0.94 & -0.9 & .22 \\
\hline 2. Más de una pareja sexual & 53.13 & -0.46 & 0.10 & 1.07 & 1.7 & 1.08 & -1.2 & .13 \\
\hline 3. NUCP5 en parejas estables & 50.00 & -0.32 & 0.10 & 0.89 & $-2.7 * *$ & 0.86 & $-2.5^{* *}$ & .32 \\
\hline 4. No haber usado preservativo en el debut sexual & 46.35 & -0.16 & 0.10 & 0.91 & $-2.1 * *$ & 0.87 & $-2.3 * *$ & .29 \\
\hline 5. Tener relaciones sexuales bajo efecto de drogas & 45.05 & -0.10 & 0.10 & 1.03 & 0.6 & 1.04 & 0.6 & .17 \\
\hline 6. NUCP5 en relaciones sexuales vaginales & 43.49 & -0.03 & 0.10 & 0.86 & $-3.3^{* *}$ & 0.82 & $-3.1 * *$ & .36 \\
\hline 7. No tener iniciativa en el uso del preservativo & 43.23 & -0.02 & 0.10 & 1.07 & 1.6 & 1.12 & 1.8 & .12 \\
\hline 8. NUCP5 en relaciones sexuales anales & 40.89 & 0.09 & 0.10 & 1.11 & $2.4^{*}$ & 1.21 & $2.9^{*}$ & .08 \\
\hline 9. $\mathrm{NUCP}^{5}$ en parejas ocasionales & 31.51 & 0.53 & 0.11 & 0.92 & -1.5 & 0.84 & $-2.0^{* *}$ & .29 \\
\hline 10. Usar la píldora del día después & 19.01 & 1.25 & 0.13 & 1.20 & $2.3^{*}$ & 1.27 & 1.7 & .02 \\
\hline
\end{tabular}

FIT: Estadístico de ajuste para evaluar la dependencia de los patrones próximos; ${ }^{2}$ MNSQ: Residuo cuadrático medio; ${ }^{3}$ ZSTD: Residuo cuadrático medio normalizado; ${ }^{4}$ OUTFIT: Estadístico de ajuste para evaluar el impacto de los patrones extremos; ${ }^{5}$ NUCP: No usar de forma consistente el preservativo.

El paso siguiente fue eliminar el ítem 8 del total (baja correlación ítem-total y falta de ajuste) y calibrar los ítems. Posteriormente, se estimó el riesgo de los participantes. Finalmente, se procedió a estimar el parámetro de riesgo de las personas (eliminando los ítems 3, 4 y 6). El porcentaje de personas con valores Outfit e Infit estandarizados no significativos para un nivel de confianza del $95 \%$ fue de 7 y 3 casos, respectivamente, indicando un buen ajuste de los patro- nes de los participantes al modelo de Rasch. El valor medio de riesgo de los participantes fue de -.39 (DT = 1.11) en unidades logits, indicando que hay mayor probabilidad de no realizar una conducta de riesgo que de realizarla. En la Figura 1 se puede observar la distribución de frecuencias de los valores de riesgo de los participantes. 
Número de adolescentes

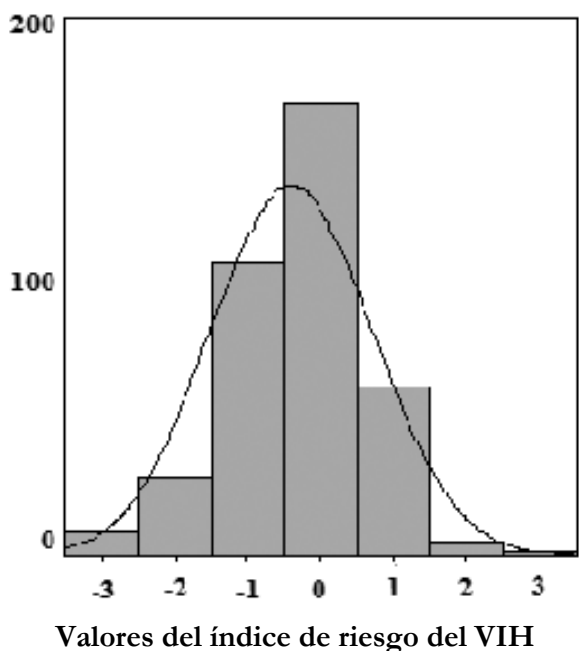

Figura 1. Distribución de frecuencias de los adolescentes índice de riesgo global del VIH construido.

\section{Diferencias en función del sexo y religiosidad en} Control sobre la Relación y Doble Moral

La media en Control sobre la Relación fue 3.09 (DT = $0.50)$, siendo $3.03(D T=0.50)$ en varones y $3.22(D T=0.46)$ en mujeres. Estas diferencias fueron estadísticamente significativas $\left(F_{1,596)}=19.20, p<.01 ; d\right.$ de Cohen $\left.=.38\right)$.

La media en Doble Moral fue $3.02(D T=0.92)$, siendo $3.24(D T=0.86)$ en varones y $2.58(D T=0.89)$ en mujeres. Estas diferencias fueron estadísticamente significativas $\left(F_{(1}\right.$, ${ }_{595)}=76.85, p<.01 ; d$ de Cohen $=.76$ ).

La correlación entre Control sobre la Relación y Doble Moral fue de $-.22(p<.01)$, siendo -.13 $(p<.05)$ en varones $y$ $-.25(p<.01)$ en mujeres.

El ser practicante de una religión no tuvo efecto sobre el Control sobre la Relación $\left(F_{(1,578)}=1.26, p=.24\right)$ ni sobre la Doble Moral $\left(F_{(1,578)}=0.86, p=.42\right)$.

\section{Variables predictoras del riesgo estimado de infec-} ción por VIH

Se empleó una regresión múltiple por pasos para el grupo de varones y otra para el de mujeres para observar la influencia sobre el índice global de riesgo. Para este último grupo, el principal predictor del riesgo de infección por el VIH fue el Control sobre la Relación (véase Tabla 2); sin embargo, la Doble Moral no mejoró el modelo.

Tabla 2. Predictores del índice de riesgo de VIH en mujeres.

\begin{tabular}{lrcl}
\hline Predictores & Beta & $t(p)$ & Tolerancia \\
\hline Frecuencia relaciones anales & .168 & $2.061(.041)$ & .881 \\
Frecuencia relaciones vaginales & .184 & $2.310(.022)$ & .882 \\
Control sobre la relación & -.231 & $-3.072(.003)$ & .987 \\
\hline$R^{2}=.177$ & & &
\end{tabular}
$\mathrm{R}^{2}=.177$
En la regresión para la muestra de varones (véase la Tabla 3), el Control sobre la Relación se asocia con menores niveles de riesgo $(p<.01)$ así como la doble moral $(p=$ 0.042).

Para ambos sexos, las frecuencias de las relaciones sexuales vaginales y anales se relacionaron con el riesgo del VIH. Esta asociación fue mayor en varones $(p<.01)$. En ambos casos, la capacidad predictiva del modelo fue baja $(17.7 \%$ en varones y $15.7 \%$ en mujeres).

Tabla 3. Predictores del índice de riesgo del VIH en varones.

\begin{tabular}{lccc}
\hline Predictores & Beta & $t(p)$ & Tolerancia \\
\hline Frecuencia relaciones anales & .205 & $3.572(<.001)$ & .951 \\
Frecuencia relaciones vaginales & .228 & $3.978(<.001)$ & .957 \\
Control sobre la relación & -.148 & $-2.616(.009)$ & .982 \\
Doble moral & .115 & $2.045(.042)$ & .990 \\
\hline$R^{2}=.157$ & & &
\end{tabular}

\section{Discusión}

Los resultados de este estudio indican que las mujeres tienen un mayor control en la relación que los varones. Este dato adquiere importancia porque esta variable es la más relacionada con la protección para la infección del VIH. Es decir, cuando una mujer toma decisiones sobre sus relaciones de pareja y/o sexuales, aumenta la probabilidad de que utilice el preservativo. Estos resultados son similares a los obtenidos por Greig et al. (2008) y Pulerwitz et al. (2002), donde se concluye que tener poder en la relación implica un mayor uso del preservativo. Además, otros estudios explican el bajo uso del preservativo a partir del bajo poder en la relación de las mujeres (Fernández-Esquer et al., 2004; Harvey et al., 2002). Concretamente, aquellas mujeres con relaciones donde sus parejas tienen mayor poder y control, presentan mayor consumo de drogas (Jewkes, Dunkle, Nduna y Shai, 2010), más probabilidades de padecer, al menos, un tipo de disfunción sexual y menor satisfacción sexual (Lau et al., 2006), mayor probabilidad de suicidio (Canetto, 2008) y mayor probabilidad de ser infectadas por el VIH y otras ITS (Bermúdez, Castro, Gude et al., 2010; Herrera y Campero, 2002; Pulerwitz y Barker, 2008).

En lo que respecta a la doble moral, se ha obtenido que las mujeres puntúan menos que los varones. A su vez, la baja adherencia a la doble moral se relaciona con un mayor poder en la relación y éste predice un menor riesgo de infección por VIH. Matud, Padilla y Gutiérrez (2005) afirman que una relación de pareja igualitaria permite una mayor capacidad de decisión y negociación de los miembros, lo que contribuye a una relación más sana y a una mayor igualdad en los roles de género dentro de la misma. Además, una menor adhesión a la doble moral se relaciona con una mayor asertividad sexual (Santos-Iglesias y Sierra, 2010). En los resultados no se encuentra que la doble moral correlacione con el índice de riesgo de infección del VIH. Este dato es similar a los obtenidos en diversas investigaciones (Bermúdez, Castro, Gude et al., 2010; Kreager y Staff, 2009). 
Por otra parte, se encuentra que el grupo de varones muestra una mayor adhesión a la doble moral y, en función de ésta, tratarían de mantener relaciones sexuales desprotegidas. Se puede concluir que, en mujeres, la única variable, de las analizadas en este estudio, que predice un mayor riesgo de infección por $\mathrm{VIH}$, es el poder en la relación de pareja, tal y como encuentran otros autores (Fernández-Esquer et al., 2004; Kreager y Staff, 2009; Pulerwitz y Barker, 2008).

En el grupo de varones, la doble moral se relaciona con mayor riesgo de infección. Estos puntúan más alto en doble moral, lo que indica que mantienen las creencias de que la iniciativa y el control sexual debe ser del varón, y sobre rechazar el uso del preservativo. Resultados similares obtienen Bermúdez, Castro, Gude et al. (2010), donde los varones latinoamericanos e hispanos obtienen mayores puntuaciones que las mujeres, y Sierra y Gutiérrez-Quintanilla (2007), con muestra salvadoreña. Este hecho cobra importancia ya que, en varones, la adhesión a la ideología masculina se relaciona con una actitud negativa hacia el uso del preservativo (Noar y Morokoff, 2002). Todo ello, pone de manifiesto que las desigualdades de género persisten y afectan a la vida de varones y mujeres (Rudman y Glick, 2008). Además, los ado-

\section{Referencias}

Antón-Ruiz, F. A. y Espada, J. P. (2009). Consumo de sustancias y conductas sexuales de riesgo para la transmisión del VIH en una muestra de estudiantes universitarios. Anales de Psicología, 25, 344-350.

Baumeister, R. F. y Twenge, J. M. (2002). Cultural Suppression of Female Sexuality. Review of General Psychology, 6, 166-203.

Bermúdez, M. P., Castro, A. y Buela-Casal, G. (2009). Sida en España e inmigración: análisis epidemiológico. Universitas Psychologica, 8, 37-47.

Bermúdez, M. P., Castro, A., Gude, F. y Buela-Casal, G. (2010). Relationship power in the couple and sexual double standard as predictors of the risk of sexually transmitted infections and HIV: Multicultural and gender differences. Current HIV Research, 8, 172-178.

Bermúdez, M. P., Castro, A., Madrid, J. y Buela-Casal, G. (2010). Análisis de la conducta sexual de los adolescentes autóctonos e inmigrantes latinoamericanos en España. International Journal of Clinical and Health Psychology, 10, 89-103.

Bowleg, L., Belgrave, F. Z. y Reisen, C. A. (2000). Gender Roles, Power Strategies, and Precautionary Sexual Self-Efficacy: Implications for Black and Latina Women's HIV/AIDS Protective Behaviours, Sex Roles, 42, 613-636.

Canetto, S. S. (2008). Women and Suicidal Behavior: A Cultural Analysis. American Journal of Orthopsychiatry, 78, 259-266.

Caron, S. L., Davis, C. M., Halteman, W. A. y Stickle, M. (2003). Double Standard Scale. En C.M. Davis, W.L. Yarber, R. Bauserman, G. Schreer y S.L. Davis (Eds.), Handbook of sexuality-related measures (pp. 182-183). California: Sage Publications.

Castro, A. y Bermúdez, M. P. (2011). Native and immigrant adolescents in Spain: Adaptation and perceived discrimination as HIV-risk factors. International Journal of Clinical and Health Psychology, 11, 34-47.

Dávila, Y. R. (2002). Influence of Abuse on Condom Negotiation Among Mexican-American Women Involved in Abusive Relationships. Journal of the Association of Nurses in AIDS Care, 13, 46-56.

Eaton, D. K., Kann, L., Kinchen, S., Shanklin, S., Ross, J., Hawkins, J., Harris, W.A., Lowry, R., McManus, T., Chyen, D., Lim, C., Whittle, L., Brener, N. D. y Wechsler, H. (2010). Youth Risk Behavior Surveillance United States, 2009 (Surveillance Summaries, 59). Atlanta, EEUU: Centers for Disease Control and Prevention (CDC).

Fagan, P. y McDonell, P. (2010). Knowledge, attitudes and behaviours in relation to safe sex, sexually transmitted infections (STI) and HIV/AIDS lescentes no perciben la desigualdad de género, particularmente los varones, ya que se trata de un fenómeno a menudo invisible para aquellos que son privilegiados por él (Lott y Bullock, 2010).

Por último, no se observa relación significativa entre el control en la relación y la doble moral. Es decir, el grado de control que se tiene sobre la relación de pareja es independiente de las ideas que se tenga sobre el rol que cada uno de los sexos debe ejercer en la misma. Esto es interesante puesto que no se han encontrado investigaciones centradas en la relación de estas dos variables. Si bien diversos estudios analizan ambas (Bermúdez, Castro, Gude et al., 2010; Bowleg, 2000; Noar y Morokoff, 2002), no examinan específicamente la relación entre ellas.

En resumen, se ha comprobado la influencia de la adhesión a la doble moral en varones adolescentes, y entre el control y poder en la relación, en mujeres, y la emisión de conductas sexuales de riesgo. Futuras investigaciones deberán aclarar cómo incluir estas variables en programas de intervención y poder utilizarlas para aumentar el uso del preservativo y disminuir, con ello, el número de casos de ITS y VIH.

among remote living north Queensland youth. Australian and New Zealand Journal of Public Health, 34, 52-56.

Fernández-Esquer, M. E., Atkinson, J., Diamond, P., Useche, B. y Mendiola, R. (2004). Condom use self-efficacy among U.S. and foreign-born latinos in Texas. The Journal of Sex Research, 41, 390-399.

Gayet, C., Juárez, F., Pedrosa, L. A. y Magis, C. (2003). Uso del condón entre adolescentes mexicanos para la prevención de las infecciones de transmisión sexual. Salud Pública de México, 45, 632-640.

Greig, A., Peacock, D., Jewkes, R. y Msimang, S. (2008). Gender and AIDS: time to act. AIDS, 28, S35-S42.

Gurman, T. y Borzekowski, D. L. G. (2004). Condom use among latino college students. Journal of American College Health, 25, 169-178.

Harvey, S. M., Beckman, L.J., Browner, C. H. y Sherman, C. A. (2002). Relationship Power, Decision Making, and Sexual Relations: An Exploratory Study with Couples of Mexican Origin. The Journal of Sex Research, 39, 284-291.

Herrera, C. y Campero, L. (2002). La vulnerabilidad e invisibilidad de las mujeres ante el VIH/SIDA: constantes y cambios en el tema. Salud Pública de México, 44, 554-564.

Jackson, S. M. y Cram, F. (2003). Disrupting the sexual double standard: young women's talk about heterosexuality. British Journal of Social Psychology, 42, 113-27.

Jewkes, R., Dunkle, K., Nduna, M. y Shai, N. (2010). Intimate partner violence, relationship power inequity, and incidence of HIV infection in young women in South Africa: a cohort study. Lancet, 376, 41-48.

Kalichman, S. C., Eaton, L. y Cherry, C. (2010). Sexually transmitted infections and infectiousness beliefs among people living with HIV/AIDS: implications for HIV treatment as prevention. HIV Medicine, 11, 502509.

Kotchick, B. A., Shaffer, A., Forehand, R. y Miller, K. S. (2001). Adolescent sexual risk behavior: a multi-system perspective. Clinical Psychology Review, 21, 493-519.

Kreager, D. A. y Staff, J. (2009). The Sexual Double Standard and Adolescent Peer Acceptance. Social Psychology Quarterly, 72, 143-164.

Lau, J., Yang, X., Wang, Q., Cheng, Y., Tsui, H., Mui, H. y Kim, J. (2006). Gender and marital relationship as predictors of sexual disfunction and sexual satisfaction among younger married couples in rural China: a population based study. Adult Urology, 67, 579-585. 
Leftkowitz, E. S., Boone, T. L. y Shearer, C. L. (2004). Communication with best friends about sex-related topics during emerging adulthood. Journal of Youth and Adolescence, 33, 339-351.

Lott, B. y Bullock, H. (2010). Social Class and women's lives. Psychology of Women Quaterly, 34, 421-424.

Matud, M. P., Padilla, V. y Gutiérrez, A. B. (2005). Mujeres maltratadas por su pareja. Guía de tratamiento psicológico. Madrid: Minerva Ediciones.

Moreno-Rodríguez, D., Rivera-Paniagua, B., Robles-Montijo, S., BarrosoVillegas, R., Frias-Arroyo, B. y Rodríguez-Cervantes, M. (2008). Características del debut sexual de los adolescentes y determinantes del uso consistente del condón desde el análisis contingencial. Psicología y Salud, 18, 207-225.

Montero, I. y León, O. G. (2007). A guide for naming research studies in Psychology. International Journal of Clinical and Health Psychology, 7, 847862.

Motti-Stefanidi, F., Pavlopoulos, V., Obradovi, J., Dalla, M., Takis, N., Papathanassiou, A. y Masten, A. S. (2008). Immigration as a risk factor for adolescent adaptation in Greek urban schools. European Journal of Developmental Psychology, 5, 235-261.

Muñiz, J. M., Hidalgo, A.M., Cueto, E., Martínez, R. y Moreno, R. (2005). Análisis de los Ítems. Cuadernos de Estadística. Madrid: Editorial La Muralla.

Noar, S. M. y Morokoff, P. J. (2002). The relationship between masculinity ideology, condom attitudes, and condom use stage of change: a structural equation modelling approach. International Journal of Men's Health, 1 , 43-58.

Organista, K. C., Organista, P. B., Bola, J. R., García-de-Alba, J. E. y Castillo-Morán, M. A. (2000). Predictors of condom use in Mexican migrant labourers. American Journal of Community Psychology, 28, 245-265.

Phipps, M. G., Matteson, K. A., Fernández, G. E., Chiaverini, L. y Weitzen, S. (2008). Characteristics of women who seek emergency contraception and family planning services. American Journal of Obstetrics \& Gynecology, 199, 111.e1-111.e5.

Piña, J. A., Lozano, D. I., Vázquez, P. y Carillo, I. C. (2010). Motivos y uso de preservativo en estudiantes universitarios de Ciudad Juárez (México). Anales de Psicología, 26, 18-26.

Pulerwitz, J., Amaro, H., De Jong, W., Gortmaker, S. L. y Rudd, R. (2002). Relationship power, condom use and HIV risks among women in the USA. AIDS Care, 14, 789-800.
Pulerwitz, J. y Barker, G. (2008) Measuring attitudes toward gender norms among young men in Brazil. Development and psychometric evaluation of the GEM Scale. Men and Masculinities, 10, 322-338.

Pulerwitz, J., Gortmaker, S. L. y De Jong, W. (2000). Measuring sexual relationship power in HIV/AIDS research. Sex Roles, 42, 637-660.

Rasch, G. (1960). Probabilistic Models for Some Intelligence and Attainment Test. Chicago: University of Chicago Press.

Robles-Montijo, S., Piña-López, J.A. y Moreno-Rodríguez, D. (2006). Determinantes del uso inconsistente del condón en mujeres que tienen sexo vaginal, oral y anal. Anales de Psicología, 22, 200-204.

Rudman, L. A. y Glick, P. (2008). The social psychology of gender: how power and intimacy shape gender relations. New York: Guildford Press.

Sander, P. M., Raymond, E. G. y Weaver, M. A. (2009). Emergency contraceptive use as a marker of future risky sex, pregnancy, and sexually transmitted infection. American Journal of Obstetrics and Gynecology, 201 146.e1-146.e6

Santos-Iglesias, P. y Sierra, J. C. (2010). El papel de la asertividad sexual en la sexualidad humana: una revisión sistemática. International Journal of Clinical and Health Psychology, 10, 553-577.

Sierra, J. C. y Gutiérrez-Quintanilla, J. R. (2007). Estudio psicométrico de la versión salvadoreña de la Double Standard Scale. Cuadernos de Medicina Psicosomática y Psiquiatría de Enlace, 82, 23-30.

Teva, I., Bermúdez, M. P. y Buela-Casal, G. (2009). Variables sociodemográficas y conductas de riesgo en la infección por el VIH y las enfermedades de transmisión sexual en adolescentes. España, 2007. Revista Española de Salud Pública, 83, 309-320.

Teva, I., Bermúdez, M. P. y Buela-Casal, G. (2011). Búsqueda de sensaciones sexuales, estilos de afrontamiento, estrés social y su relación con la conducta sexual adolescente. Anales de Psicología, 27, 35-46.

Wright, B. D. y Linacre, J. M. (1988). A User's Guide to BIGSTEPS. RaschModel Computer Programs. Chicago, Illinois: MESA Press.

Wright B. D. y Masters G. N. (1982). Rating Scale Analysis. Chicago: MESA Press.

Zangao, M. O. B. y Sim-Sim, M. M. (2011). Sexual double standar and affective-sexual behaviors in adolescence. Revista de Enfermagem UFPE On Line, 5, 328-335.

(Articulo recibido: 26-9-2011; revisado: 30-10-2012; aceptado: 30-10-2012) 